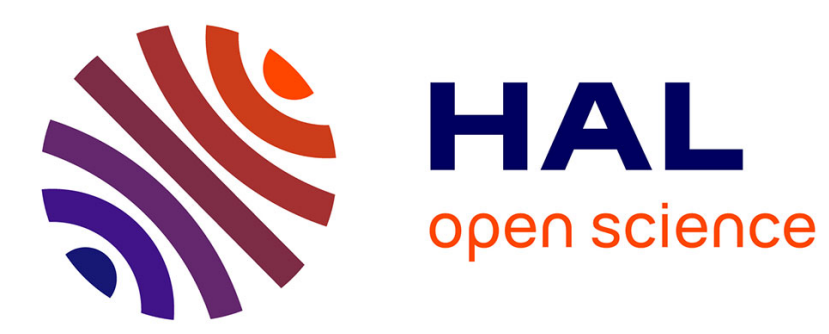

\title{
Recent Advances in Multiresolution Analysis of 3D Meshes and their Applications
}

Michael Roy, Sebti Foufou, Frederic Truchetet

\section{To cite this version:}

Michael Roy, Sebti Foufou, Frederic Truchetet. Recent Advances in Multiresolution Analysis of 3D Meshes and their Applications. Wavelet Applications in Industrial Processing, Jan 2010, San Jose, United States. 10.1117/12.845595 . hal-01959348

\section{HAL Id: hal-01959348 https://hal.science/hal-01959348}

Submitted on 18 Dec 2018

HAL is a multi-disciplinary open access archive for the deposit and dissemination of scientific research documents, whether they are published or not. The documents may come from teaching and research institutions in France or abroad, or from public or private research centers.
L'archive ouverte pluridisciplinaire HAL, est destinée au dépôt et à la diffusion de documents scientifiques de niveau recherche, publiés ou non, émanant des établissements d'enseignement et de recherche français ou étrangers, des laboratoires publics ou privés. 


\title{
Recent Advances in Multiresolution Analysis of 3D Meshes and their Applications
}

\author{
Michaël Roy, Sebti Foufou, Frédéric Truchetet \\ Le2i - Laboratoire d'Électronique, Informatique et Image \\ CNRS UMR 5158 - Université de Bourgogne \\ Route des Plaines de L'Yonne - 89000 Auxerre - France
}

\begin{abstract}
3D meshes are widely used in computer graphics applications for approximating 3D models. When representing complex shapes in raw data format, meshes consume a large amount of space. Applications calling for compact and fast processing of large 3D meshes have motivated a multitude of algorithms developped to process these datasets efficiently. The concept of multiresolution analysis proposes an efficient and versatile tool for digital geometric processing allowing for numerous applications. In this paper, we survey recent developments in multiresolution methods for 3D triangle meshes. We also show some results of these methods through various applications.
\end{abstract}

Keywords: Multiresolution analysis, levels of detail, 3D triangle mesh, applications

\section{INTRODUCTION}

3D computer graphics have been more and more used in different scientific and artistic fields, such as education, science, engineering, medecine, advertising, entertainment, etc. This implies the use of 3D models through different kinds of representation : explicit with triangle meshes, parametric with splines, and implicit with functions. These 3D models are more and more precise and complex, and can be obtained from various sources: modeling software, acquisition device, scientific simulation. In the last decades, the processing of 3D models has emerged as an active and very productive research area. The need to process high-quality 3D models has driven the research on efficient data structures and algorithms. In this paper, we only consider 3D models represented with triangle meshes.

Multiresolution techniques have proven their efficiency through a large number of applications: compression, feature extraction, rendering, modeling, filtering, etc. Moreover these techniques have been applied on different kinds of data, such as signals, images, meshes, etc. Multiresolution techniques decompose the initial data into a hierarchy that represents different levels of detail. Technically this hierarchy can be exploited in order to significantly speed up many algorithms. More important, however, is the semantic effect in that such hierarchies can be used for intuitive modeling metaphors or highly efficient geometry compression algorithms. An example of the multiresolution decomposition of a mesh is shown in Figure 1. Many papers present the multiresolution analysis and the wavelet transform in the computer graphics domain. Here we refer to ${ }^{1-4}$ as an introduction.

In this paper, we present a survey of the different multiresolution methods proposed for triangle meshes. We start with the preliminary background on triangle meshes and multiresolution analysis. Then we show the different proposed methods and their applications. We conclude this survey on the use of multiresolution methods in $3 \mathrm{D}$ computer graphics.

Author Emails: michael.roy@u-bourgogne.fr, sebti.foufou@u-bourgogne.fr, frederic.truchetet@u-bourgogne.fr 


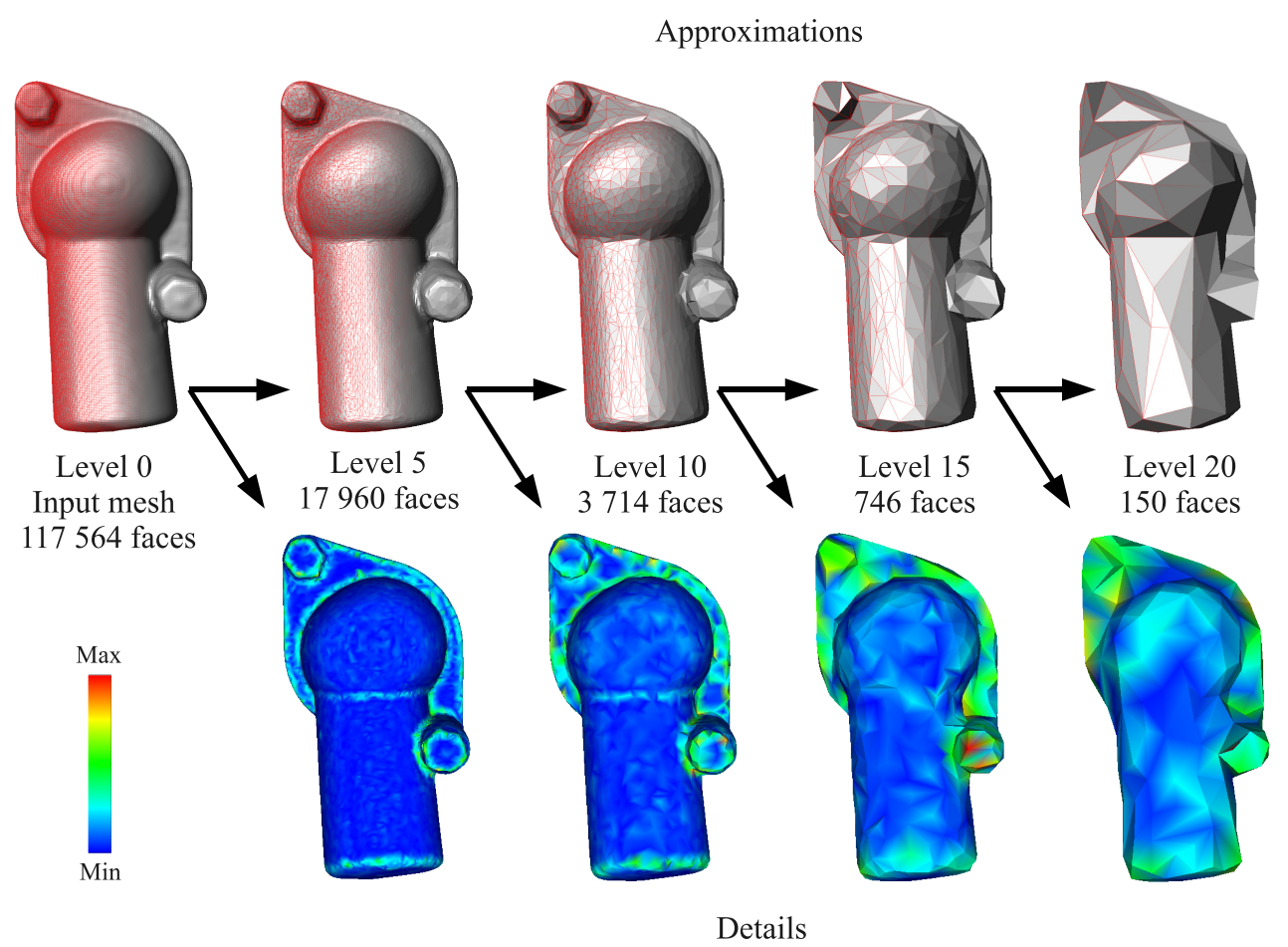

Figure 1. An example of the multiresolution decomposition of a triangle mesh. The initial mesh is decomposed in a set of levels of detail represented by an approximation mesh and detail coefficients shown in pseudo-colors. The detail coefficients represent the data lost during the appromixation process. The initial mesh can be recontructed using the approximations and the detail coefficients.

\section{BACKGROUND}

In this section, we present the necessary background for the survey proposed in this article. First, we will define how 3D models are represented using triangle meshes. We will make a difference between a semi-regular mesh and an irregular mesh. Then, we will define the multiresolution analysis originally proposed in the signal processing field.

\subsection{Triangle meshes}

$3 \mathrm{D}$ models can de represented in many different ways ${ }^{5}$; the most common representations are: polygon mesh surfaces, parametric surfaces, and implicit surfaces. Here, we only consider triangle meshes representing a 2manifolds with boundaries. We denote a triangle mesh as a pair $(P, K)$, where $P$ is a set of $N$ point positions $p_{a}=\left(x_{a}, y_{a}, z_{a}\right) \in R^{3}$ with $1 \leq a \leq N$, and $K$ is an abstract simplicial complex which contains all the topological, i.e. adjacency information. The complex $K$ is a set of subsets of $\{1, \ldots, N\}$. These subsets are called simplices and come in 3 types: vertices $v=\{a\} \in V \subset K$, edges $e=\{a, b\} \in E \subset K$, and faces $f=\{a, b, c\} \in F \subset K$, so that any non-empty subset of a simplex of $K$ is again a simplex of $K, e . g$., if a face is present so are its edges and vertices. The performances of a geometry processing application are directly influenced by the underlying triangle mesh representation and its data structure. ${ }^{3,6}$

Guskov et al. ${ }^{7}$ distinguish three types of topology among triangulations (see Figure 2). A regular mesh has vertices of degree six. An irregular mesh has vertices of any degree. A semi-regular mesh are obtained using a subdivision algorithm where coarse vertices have arbitrary degree while all other vertices have degree six. Using this terminology, we distinguish multiresolution methods for semi-regular meshes, and multiresolution methods for irregular meshes. The first ones are usually based on subdivision algorithms, and the latter on decimation algorithms. 


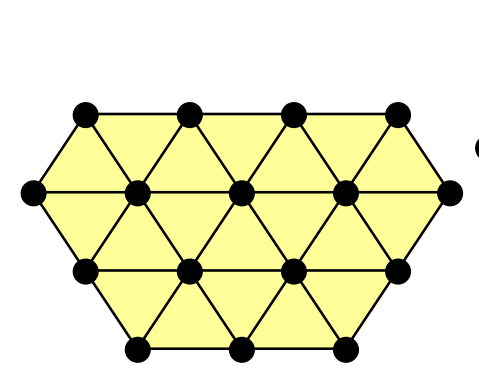

(a) Regular

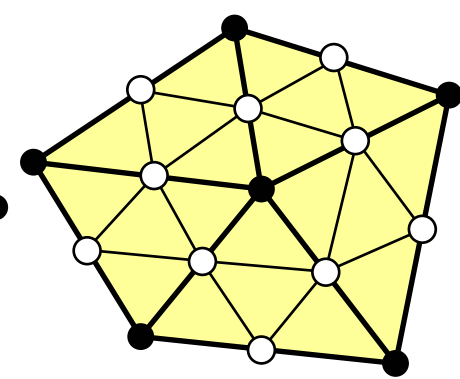

(b) Semi-regular

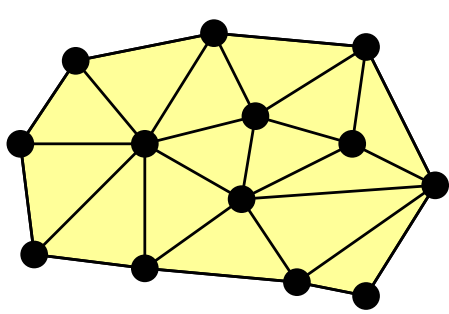

(c) Irregular

Figure 2. Different types of topology of triangle meshes. Semi-regular meshes involve the use of subdivision algorithms.

\subsection{Multiresolution methods}

Multiresolution analysis ${ }^{8-10}$ is a solid and efficient framework to represent a data set at different levels of resolution. It decomposes an initial data set into a sequence of approximations and details by projecting the data onto two sets of basis functions: the scalar and wavelet bases. The approximations are computed by using the scalar functions, and represent the initial data at different levels of resolution. The details are computed by using the wavelet functions, and encode the data lost by the approximations. The initial data can be reconstructed from the wavelet representation using the dual scalar and wavelet bases. This technique has been extended to multidimensional functions efficiently using the concept of a tensor product: the one-dimensional wavelet transform is applied to each dimension of the data independently.

Different types of basis functions are available in the literature. Every basis function has different properties, and it is best suited to solve a particular problem. Properties are the orthogonality, the size of the support, the number of vanishing moments, the symetry and the smoothness of the basis function. The main advantage of the multiresolution analysis is its representational and computational efficiency. The appeal of multiresolution methods comes from their ability to represent features of a data set through different scales.

The lifting scheme ${ }^{11}$ allows the construction of filter banks entirely in the spatial domain. Instead of explicitly specifying scaling functions and wavelets, the decomposition process is made up of so-called splitting, prediction, update, scaling and merging steps that are arranged in a flow chart (see Figure 3). Using the lifting scheme, the wavelet decomposition can be performed in-place and in linear time.

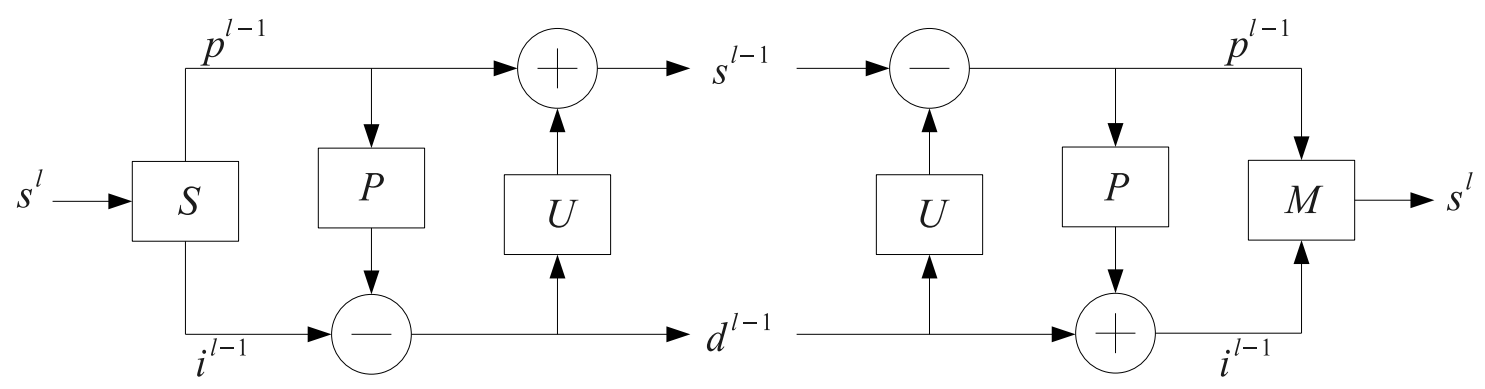

Figure 3. The lifting scheme decomposition and reconstruction process.

The multiresolution methods for 3D meshes presented in this paper do not always respect the strict definition of the wavelet transform. Some methods use so-called pyramid algorithms, whose construction is very close to the wavelet transform. In fact, multiresolution techniques on semi-regular meshes use subdivision schemes, which allow the construction of wavelets. Multiresolution techniques on irregular meshes do not always have this guarantee, but sometimes it can be achieved. 


\section{MULTIRESOLUTION DECOMPOSITION OF SEMI-REGULAR MESHES}

In this section, we present the main multiresolution methods for semi-regular meshes. Lounsbery ${ }^{12}$ made the connection between wavelet analysis and subdivision methods to define different levels of resolution. This technique called subdivision wavelet transform uses the theory of the multiresolution analysis and of the subdivision rules to construct a multiresolution surface representation for surface with subdivision connectivity (i.e. semiregular mesh). In the Lounsbery decomposition, a mesh is subdivided using Loop subdivision, ${ }^{13}$ and deformed to make it fit the surface to be approximated. These steps can be repeated depending on the required resolution levels. Multiresolution analysis is computed with two analysis filters, $A^{j}$ and $B^{j}$ for each resolution level $j$. The reconstruction is done with two synthesis filters $P^{j}$ and $Q^{j}$. These filters are represented using matrix notation and, to ensure exact reconstruction, must satisfy the following constraint:

$$
\left(\begin{array}{l}
A^{j} \\
B^{j}
\end{array}\right)=\left(\begin{array}{ll}
P^{j} & Q^{j}
\end{array}\right)^{-1},
$$

Let us call $V^{j}$ the vertex positions of the mesh at the level $j$ and $W^{j}$ the corresponding wavelet coefficients, the decomposition is computed as:

$$
\begin{aligned}
& V^{j}=A^{j+1} V^{j+1}, \\
& W^{j}=B^{j+1} V^{j+1} .
\end{aligned}
$$

The reconstruction is made using the following relation:

$$
V^{j+1}=P^{j} V^{j}+Q^{j} W^{j} .
$$

The decomposition process is shown on Figure 4.

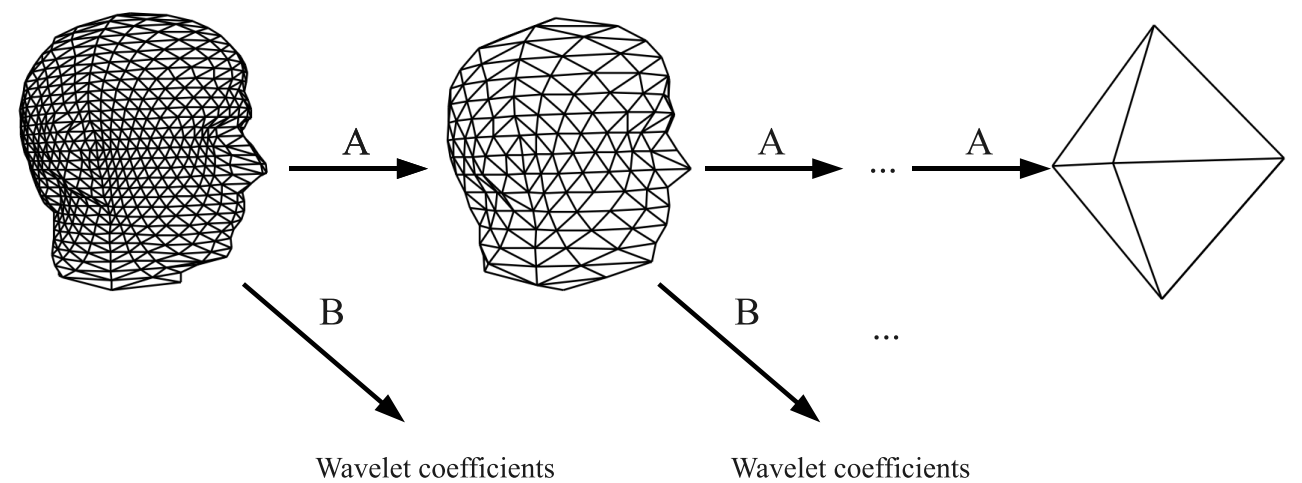

Figure 4 . The Lounsbery decomposition process. ${ }^{12}$

Schröder and Sweldens ${ }^{14}$ defined the spherical wavelets using Lounsbery decomposition and the lifting scheme. They show how to construct biorthogonal wavelets for scalar functions defined on a triangulated sphere. The authors experiment different vertex bases that are easy to implement and allow fully adaptive subdivision. They give examples of application with topograhic data and bidirectional reflection distribution functions. Bonneau ${ }^{15}$ improved the orthogonalization of the decomposition of spherical data with optimal triangular Haar bases.

Zorin et al. ${ }^{16}$ proposed a combination of subdivision and smoothing algorithms to construct a set of algorithms for interactive multiresolution editing of complex meshes with arbitrary topology with no attributes. Loop subdivision is used for the estimation of the high resolution mesh from the coarse representation. The authors introduce Taubin smoothing operation ${ }^{17}$ in the transformation and use a Burt-Adelson pyramid scheme ${ }^{18}$ (see Figure 5). They demonstrate the efficiency of their method through an adaptive editing and rendering application. 


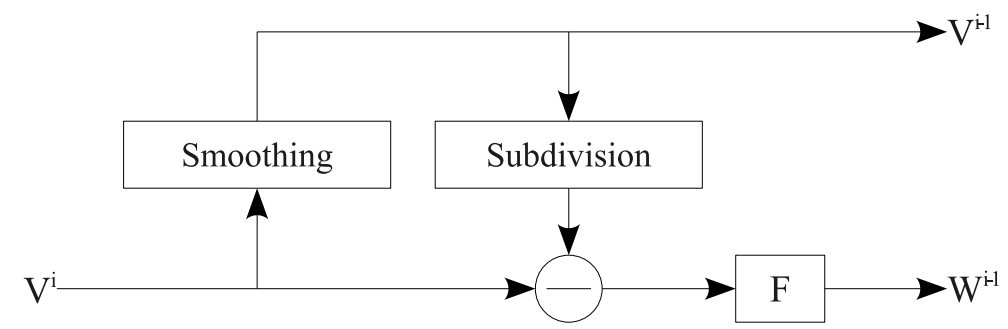

Figure 5. The Burt-Adelson pyramid used in the Zorin multiresolution decomposition.

Khodakovsky et al. ${ }^{19}$ use MAPS parametrization ${ }^{20}$ and propose a novel Loop wavelet transform. They introduce a zero-tree coder to achieve mesh compression with very good results.

Bertram $^{21}$ proposed a biorthogonal Loop-subdivision wavelet scheme. Wavelets are orthogonalized with respect to local scaling functions. This way, the wavelet analysis computes locally a least squares fit when reducing the resolution and converting geometric detail into sparse wavelet coefficients. The contribution of Bertram approach is local computation of both, wavelet analysis and synthesis in linear time.

Wang et al. ${ }^{22}$ proposed an new biorthogonal wavelet decomposition of semi-regular meshes using the $\sqrt{3}$ subdivision. ${ }^{23}$ The $\sqrt{3}$-subdivision has a slower topological refinement than the traditional triangular subdivisions, and allows the multiresolution analysis to be more balanced than the existing wavelet analyses on triangular meshes. The $\sqrt{3}$-subdivision based wavelet decomposition offers more levels of detail for processing polygonal models with good performances.

Recently, Roudet et al..$^{24,25}$ proposed a new patch-based multiresolution analysis of semi-regular mesh surfaces. They proposed a mesh partitioning method relying on surface roughness (based on frequency magnitude variations), in order to produce patches, representative of semantic attributes of the object. The partitioning framework uses a segmentation algorithm based on the wavelet coefficient magnitude, which every remeshing or compressing algorithm tends to minimize. Each produced partition share surface roughness homogeneity, inherently reflected by wavelet coefficient magnitude. The authors show the efficiency of this patch-based decomposition with a progressive compression application.

All of the previous methods are designed for semi-regular meshes, so you need to have triangle meshes with subdivision connectivities. If you have irregular meshes as input and if you want to use one of the previous methods, you can remesh the surface of the 3D model using one of the several methods proposed in the literature. ${ }^{20,26-29}$

\section{MULTIRESOLUTION DECOMPOSITION OF IRREGULAR MESHES}

Different approaches have been presented in order to deal directly with irregular meshes. This has the advantage of avoiding the remeshing process, but you loose the support of the subdivision framework.

Bonneau ${ }^{30}$ introduced the concept of multiresolution analysis over non-nested spaces, which are generated by the so-called BLaC-wavelets, a combination of the Haar function with the linear B-Spline function. This concept was then used to construct a multiresolution analysis over irregular meshes. Two operators are introduced: a smoothing operator to compute the coarse mesh, and an error operator to determine the difference between the approximation mesh and the initial one. Bonneau uses a hierarchical Delaunay triangulation to encode the decomposition.

Kobbelt $\mathrm{et} \mathrm{al.}{ }^{31}$ proposed a multiresolution editing tool for irregular meshes using the discrete fairing method. The authors use the progressive mesh algorithm ${ }^{32}$ to build the coarse resolution mesh, and a smoothing operator to estimate the high resolution mesh. This method is not a wavelet transform in the strict sense, but it gives good ideas to construct multiresolution mesh using a decimation algorithm and a smoothing operator. 
Daubechies $^{33}$ and Guskov ${ }^{7}$ presented a series of non-uniform signal processing algorithms designed for irregular triangulation. They used a smoothing algorithm combined with existing hierarchical methods to build subdivision, pyramid, and wavelet algorithms for meshes with irregular connectivity. The authors proposed a non-uniform subdivision to build a geometrically smooth mesh with the same connectivity as the original mesh.

Roy et al..$^{34,35}$ proposed a novel multiresolution decomposition based on Guskov method. They use global decimation method based on the quadric error metric ${ }^{36}$ and the Meyer smoothing operator ${ }^{37,38}$ as predict operator to compute detail coefficients. Also, they extend the multiresolution analysis to appearance attributes such as vertex colors and normal vectors. The authors show experimental results through denoising and adaptive rendering applications.

Valette et al. ${ }^{39}$ presented a new wavelet-based multiresolution analysis of irregular surface meshes. This method is based on Lounsbery decomposition. The authors proposed a new irregular subdivision scheme, which allows the algorithm to be applied directly to irregular meshes. They use three codebooks to describe the different merge-split cases, and define some constraints in order to keep the simplification/decomposition step reversible. The method is a fine-to-coarse decomposition, and uses a complex simplification algorithm in order to define surface patches suitable for the irregular subdivision.

Recently, Szczesna ${ }^{40,41}$ proposed a multiresolution decomposition for irregular meshes using the lifting scheme. She introduces a new predict operator using Voronoi cells in a local neighborhood.

\section{APPLICATIONS OF MULTIRESOLUTION MESHES}

The multiresolution analysis is a versatile tool and can offer a wide range of applications. The main advantages of the multiresolution analysis are: multi-scale representation of a data set, efficient encoding of the geometrical features, and fast computation. In this section, we present the main applications of multiresolution methods for triangle meshes in the computer graphics domain.

\subsection{Feature extraction}

One recent application of multiresolution meshes is the feature extraction. ${ }^{42,43}$ By using the detail coefficients through the different scales, important features (such as sharp edges) can be efficiently found. Figure 6 shows an example of sharp edge detection using segmentation of the detail coefficients.

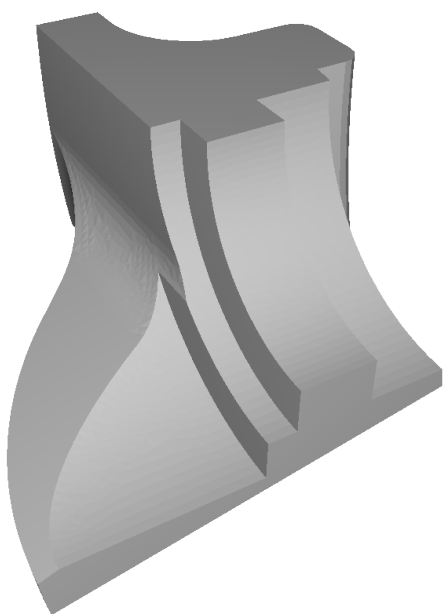

(a) Initial mesh

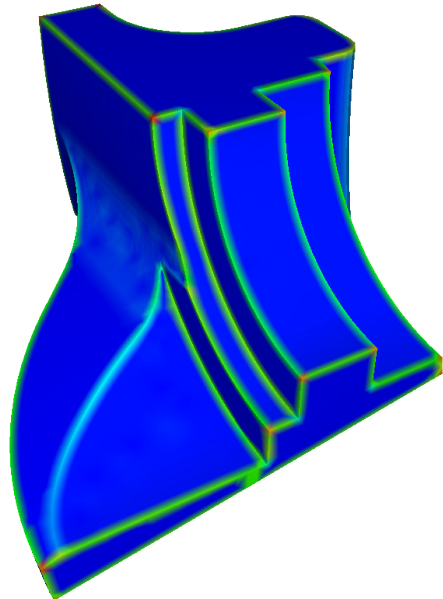

(b) Detail coefficients

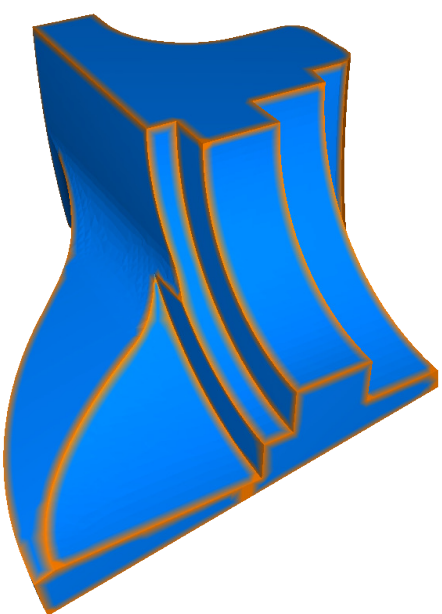

(c) Segmentation

Figure 6. An example of sharp edge extraction using segmentation of the detail coefficients from the multiresolution analysis. 
Roudet et al. ${ }^{24}$ proposed a mesh segmentation method based on the multiresolution decomposition. They use the wavelet coefficients norm and polar angle (the angle between each coefficient and its corresponding surface normal vector) obtained by the various schemes to differentiate mesh regions in term of roughness. They efficiently use this segmentation method to create patch-based multiresolution analysis and mesh compression.

\subsection{Denoising}

One of the properties of multiresolution analysis is the ability to represent a data set in a space/frequency domain. Even if the term frequency is not well defined for triangle mesh, multiresolution analysis can successfully be used to denoised meshes. ${ }^{44,45}$ Actually, most of traditional multiresolution denoising techniques can be extended to multiresolution meshes. Figure 7 shows an example of mesh denoising using soft-thresholding of the detail coefficients. ${ }^{46}$ First, the initial noisy mesh is decomposed using an irregular mesh multiresolution analysis ${ }^{34}$ and the noise is removed using a wavelet shrinkage method. The idea is to transform the data into the wavelet basis, where the large coefficients are mainly the signal, and smaller ones represent the noise. By suitably modifying these coefficients, the noise can be removed from the data. A threshold is apply to a filter operator in order to shrink the detail coefficients before the reconstruction. The soft thresholding is a fast, efficient denoising method and gives good results. Moreover, this is a non-iterative method, and so it requires only one pass over the model.

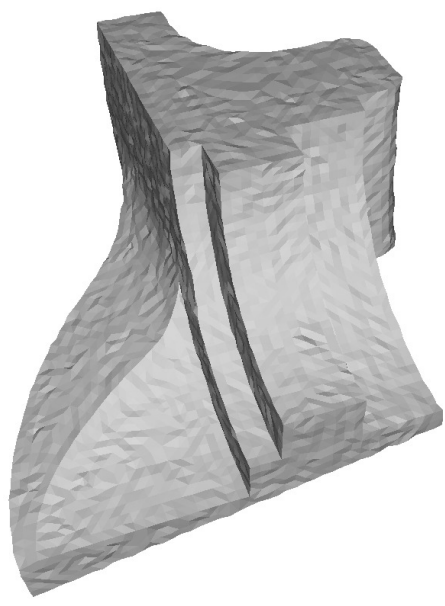

(a) Noisy mesh $\left(\sigma_{n}^{2}=10\right)$

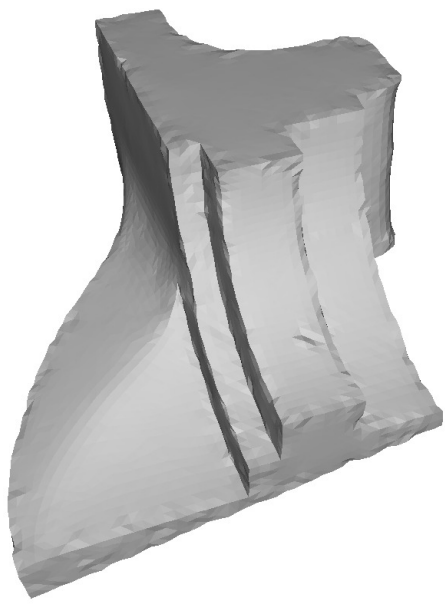

(d) Denoised mesh $\left(\sigma_{n}^{2}=10\right)$

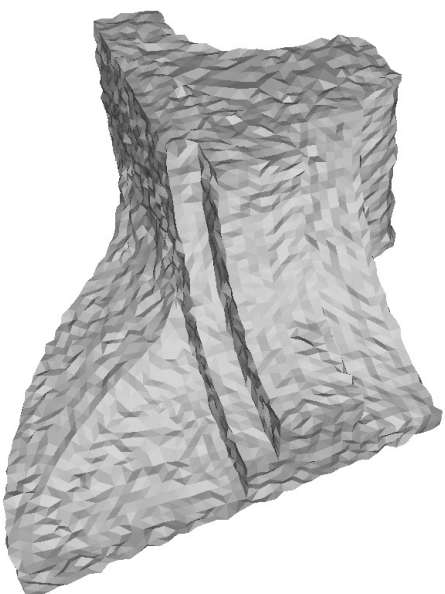

(b) Noisy mesh $\left(\sigma_{n}^{2}=25\right)$

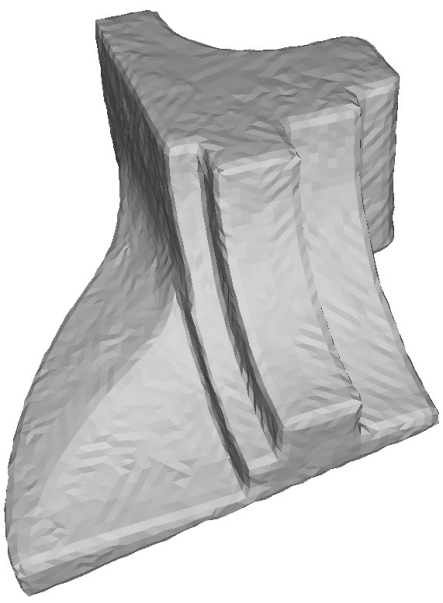

(e) Denoised mesh $\left(\sigma_{n}^{2}=25\right)$

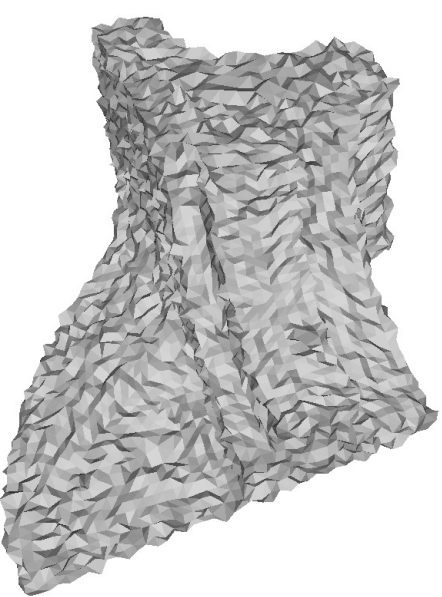

(c) Noisy mesh $\left(\sigma_{n}^{2}=50\right)$

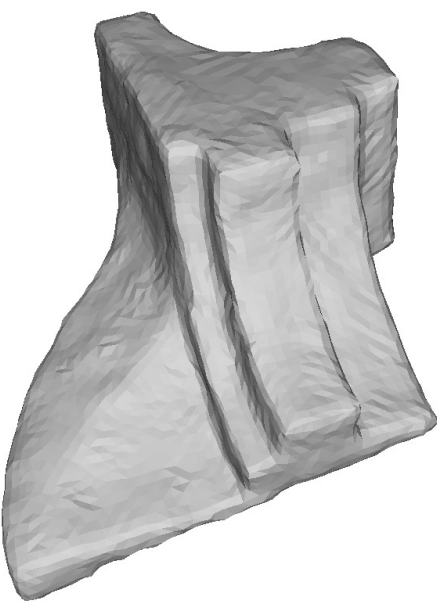

(f) Denoised mesh $\left(\sigma_{n}^{2}=50\right)$

Figure 7. An example of multiresolution mesh denoising using soft thresholding. 


\subsection{Visualization}

Multiresolution meshes offer an intrinsec hierarchical structure that can be used to developp fast, adaptive levelof-detail visualization applications. ${ }^{35,47}$ By using the detail coefficient magnitude as a vertex selection criterion, it is possible to build a view-dependent visualization with important feature preservation. Figure 8 shows an example of view-dependent visualization of a 3D multiresolution mesh. Vertices out of the viewpoint are removed using visibility criteria. Important geometrical features are preserved by thresholding the detail coefficients. If the detail coefficient length of a vertex is below a given threshold, the vertex is declared as irrelevant and thus is removed from the mesh. Moreover, different methods manage appearance attributes such as vertex colors and normal vectors. ${ }^{30,35}$

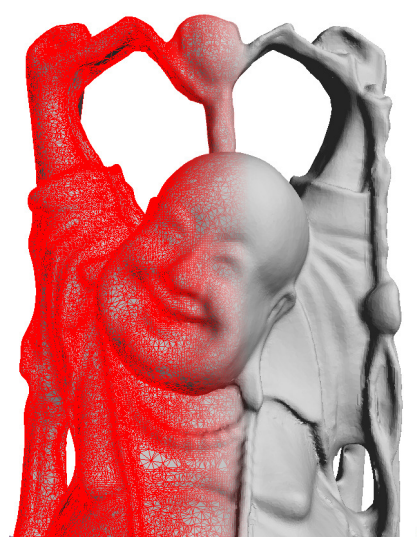

(a) $\tau=0$ (102 246 vertices)

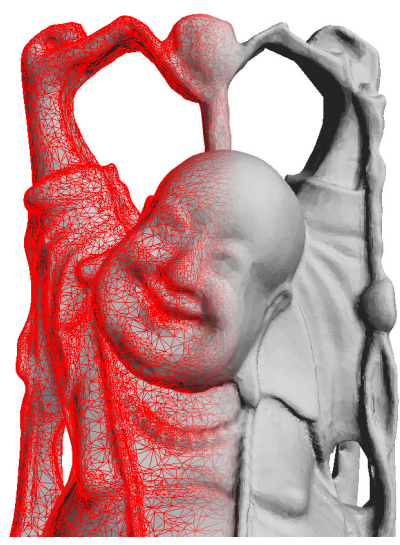

(b) $\tau=0.0001$

(31 848 vertices)

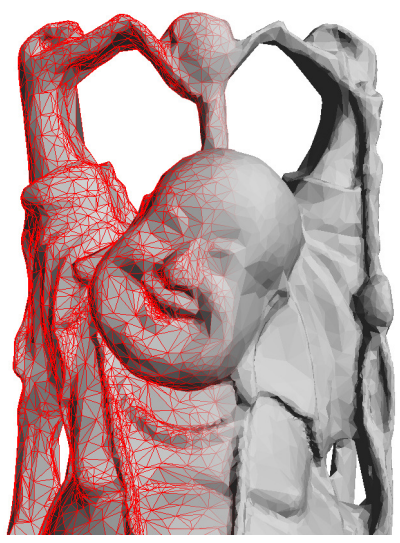

(c) $\tau=0.0004$

(9 293 vertices)

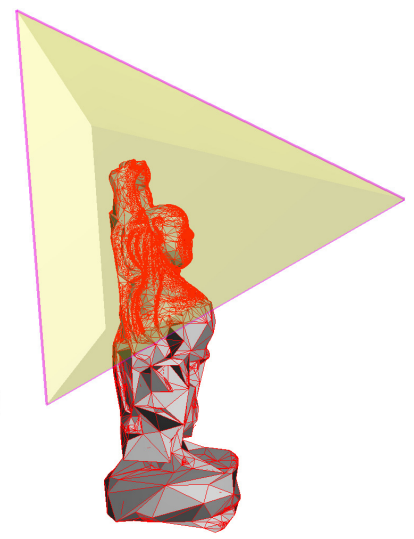

(d) $\tau=0.0004$ (viewpoint)

Figure 8. View-dependent visualization of a 3D multiresolution mesh. Important geometrical features are preserved by thresholding the detail coefficients. The initial mesh is rendered from the viewpoint in (d) with different values of the threshold $\tau$ in (a)-(c).

\subsection{Editing}

One of the first proposed applications of multiresolution meshes was modeling and editing. The idea is to edit the mesh at a lower resolution, and use the reconstruction algorithm to retrieve the high resolution mesh through its edited version. The main advantage of these methods is the ability to edit very large meshes. Applications such as industrial and engineering design require creation and manipulation of detailed geometric models. Such models are often created with 3D range sensing devices such as laser scanners, and like real world geometry, these models can carry details at many scales. Usually, multiresolution editing algorithms use local coordinate frames to parametrize the details to avoid artefacts through the different resolutions after the editing. Here, we refer to the following papers ${ }^{7,16,31,48-50}$ for more informations about multiresolution mesh modeling and editing.

\subsection{Compression}

Compression and transmission are popular applications of multiresolution meshes. Generally, mesh sequences obtained by 3D scanners or mesh design tools require huge capacity or enormous bandwidth to be stored or transmitted. For that reason, it has become an important issue to develop efficient compression methods for $3 \mathrm{D}$ mesh sequences. In multiresolution mesh compression techniques, wavelet coefficients of the analysed mesh are encoded using zerotree and entropy coding. It is also possible to used multiresolution mesh for progressive transmission over networks. Here, we refer to the following papers ${ }^{19,25,51-54}$ for more informations about multiresolution mesh compression and transmission. 


\subsection{Watermarking}

Watermarking is a recent application of multiresolution meshes. ${ }^{55}$ Digital watermarking has been considered as a potential efficient solution for copyright protection of various multimedia contents. This technique carefully hides some secret information in the cover content. Cho et al. ${ }^{56}$ proposed a fragile algorithm in the wavelet domain to authenticate semi-regular meshes. They first apply several wavelet decompositions on the original triangular mesh and then consider the facets in the obtained coarser mesh as authentication primitives. The basic idea is to slightly modify each facet so that the values of two predefined functions are the same, in order to make all these facets valid for authentication.

Recently, Wang et al..$^{57,58}$ have described a fragile watermarking technique for authenticating semi-regular meshes that is both robust to vertex reordering and similarity transformations, and capable of precisely locating the endured attacks. In their method, after one wavelet decomposition, the norm and the orientation of each obtained wavelet coefficient vector are independently modified so as to make them both imply a same watermark symbol, serving for authentication.

\section{CONCLUSION}

3D meshes being used in a wide range of fields, it is crucial to have good representation structures and efficient processing algorithms. Multiresolution analysis provides all of these in an elegant framework. In this paper, we surveyed the main methods of multiresolution analysis dedicated to triangle meshes. It is important to distinguish methods for semi-regular meshes, and methods for irregular meshes. The former requires a subdivision framework over the mesh, while the latter try to deal with irregular connectivity.

Many applications of multiresolution meshes have been proposed, such as feature extraction, denoising, editing, rendering, compression, etc. All of these applications try to give good performances using the multiresolution framework. These applications show the ability of the multiresolution analysis to efficiently represent and process heterogeneous data sets.

There is still room to research on multiresolution analysis for meshes. No mathematical framework has been created to unify them all. Moreover, many mathematical properties have yet to be highlighted or even demonstrated. But the wide number of applications show that it is still an active topic of research.

\section{REFERENCES}

[1] Stollnitz, E. J., DeRose, T. D., and Salesin, D. H., [Wavelets for Computer Graphics: Theory and Applications], Morgan Kaufmann (1996).

[2] Iske, A., Quak, E., and Floater, M. S., [Tutorials on multiresolution in geometric modelling], Springer (2002).

[3] Luebke, D., Reddy, M., Cohen, J. D., Varshney, A., Watson, B., and Huebner, R., [Level of Details for 3D Graphics], Morgan Kaufmann Publishers (2003).

[4] Dodgson, N. A., Floater, M. S., and Sabin, M. A., [Advances in Multiresolution for Geometric Modelling], Springer (2004).

[5] Foley, J., Dam, A. V., Feiner, S., and Hughes, J., [Computer Graphics: Principle and Pratice], AddisonWesley, second ed. (1990).

[6] Hubeli, A. and Gross, M., "A survey of surface representations for geometric modeling," Tech. Rep. 335, ETH Zurich, Computer Science Departement (2000).

[7] Guskov, I., Sweldens, W., and Schröder, P., "Multiresolution signal processing for meshes," in [Proceedings of ACM SIGGRAPH], 325-334 (1999).

[8] Mallat, S., "A theory for multiresolution signal decomposition : the wavelet representation," IEEE Transaction on Pattern Analysis and Machine Intelligence 11(7), 674-693 (1989).

[9] Mallat, S., [A Wavelet Tour of Signal Processing], Academic Press, second ed. (1999).

[10] Daubechies, I., [Ten Lectures on Wavelets], SIAM, Philadelphia (1992).

[11] Sweldens, W., "The lifting scheme: A construction of second generation wavelets," SIAM Journal on Mathematical Analysis 29(2), 511-546 (1998). 
[12] Lounsbery, M., DeRose, T., and Warren, J., "Multiresolution analysis for surfaces of arbitrary topological type," ACM Transactions on Graphics 16(1), 34-73 (1997).

[13] Loop, C., Smooth subdivision surfaces based on triangles, Master's thesis, University of Utah (1987).

[14] Schröder, P. and Sweldens, W., "Spherical wavelets: efficiently representing function on the sphere," in [Proceedings of ACM SIGGRAPH], 161-172 (1995).

[15] Bonneau, G.-P., "Optimal triangular haar bases for spherical data," in [Proceedings of IEEE Visualization], 279-284 (1999).

[16] Zorin, D., Schröder, P., and Sweldens, W., "Interactive multiresolution mesh editing," in [Proceedings of ACM SIGGRAPH], 259-269 (1997).

[17] Taubin, G., "A signal processing approach to fair surface design," in [Proceedings of ACM SIGGRAPH], 351-358 (1995).

[18] Burt, P. and Adelson, E., "The laplacian pyramid as a compact image code," IEEE Transactions on Communications 31(4), 532-540 (1983).

[19] Khodakovsky, A., Schröder, P., and Sweldens, W., "Progressive geometry compression," in [Proceedings of ACM SIGGRAPH], 271-278 (2000).

[20] Lee, A., Sweldens, W., Schröder, P., Cowsar, L., and Dobkin, D., "MAPS: Multiresolution adaptive parameterization of surfaces," in [Proceedings of ACM SIGGRAPH], 95-104 (1998).

[21] Bertram, M., "Biorthogonal loop-subdivision wavelets," Computing 72(1-2), 29-39 (2004).

[22] Wang, H., Qin, K., and Sun, H., " $\sqrt{3}$-subdivision-based biorthogonal wavelets," IEEE Transactions on Visualization and Computer Graphics 13(5), 914-925 (2007).

[23] Kobbelt, L., " $\sqrt{3}$-subdivision," in [Proceedings of ACM SIGGRAPH], 105-114 (2000).

[24] Roudet, C., Dupont, F., and Baskurt, A., "Multiresolution mesh segmentation based on surface roughness and wavelet analysis," in [Proceedings of SPIE Visual Communications and Image Processing], 6508 (2007).

[25] Roudet, C., Dupont, F., and Baskurt, A., "Semi-regular 3d mesh progressive compression and transmission based on an adaptive wavelet decomposition," in [Proceedings of SPIE Electronic Imaging - Wavelet Applications in Industrial Processing], 7248 (2009).

[26] Eck, M., DeRose, T., Duchamp, T., Hoppe, H., Lounsbery, M., and Stuelze, W., "Multiresolution analysis of arbitrary meshes," in [Proceedings of ACM SIGGRAPH], 173-183 (1995).

[27] Kobbelt, L., Vorsatz, J., Labsik, U., and Seidel, H.-P., "A shrink wrapping approach to remeshing polygonal surface," in [Proceedings of Eurographics], 119-130 (1999).

[28] Desbrun, M., Meyer, M., and Alliez, P., "Intrinsic parameterizations of surface meshes," in [Proceedings of Eurographics], (2002).

[29] Alliez, P., Cohen-Steiner, D., Devillers, O., Levy, B., and Desbrun, M., "Anisotropic polygonal remeshing," in [Proceedings of ACM SIGGRAPH], (2003).

[30] Bonneau, G.-P., "Multiresolution analysis on irregular surface meshes," IEEE Transactions on Visualization and Computer Graphics 4(4), 365-378 (1998).

[31] Kobbelt, L., Campagna, S., Vorsatz, J., and Seidel, H.-P., "Interactive multi-resolution modeling on arbitrary meshes," in [Proceedings of ACM SIGGRAPH], 105-114 (1998).

[32] Hoppe, H., "Progressive meshes," in [Proceedings of ACM SIGGRAPH], 99-108 (1996).

[33] Daubechies, I., Guskov, I., Schröder, P., and Sweldens, W., "Wavelets on irregular point sets," Philosophical Transactions of the Royal Society 357(1760), 2397-2413 (1999).

[34] Roy, M., Foufou, S., Koschan, A., Truchetet, F., and Abidi, M., "Multiresolution analysis for irregular meshes," in [Proceedings of SPIE Photonics East, Wavelet Applications in Industrial Processing], 5266, 249-259 (2003).

[35] Roy, M., Foufou, S., Koschan, A., Truchetet, F., and Abidi, M., "Multiresolution analysis for meshes with appearance attributes," in [Proceedings of IEEE International Conference on Image Processing (ICIP'05)], 816-819 (2005).

[36] Garland, M. and Heckbert, P., "Surface simplification using quadric error metrics," in [Proceedings of ACM SIGGRAPH], 209-216 (1997).

[37] Meyer, M., Desbrun, M., Schröder, P., and Barr, A., "Discrete differential-geometry operators for triangulated 2-manifolds," in [Proceedings of Visualization and Mathematics], (2002). 
[38] Desbrun, M., Meyer, M., Schröder, P., and Barr, A., "Implicit fairing of irregular meshes using diffusion and curvature flow," in [Proceedings of ACM SIGGRAPH], 317-324 (1999).

[39] Valette, S. and Prost, R., "Wavelet-based multiresolution analysis of irregular surface meshes," IEEE Transactions on Visualization and Computer Graphics 10(2), 113-122 (2004).

[40] Szczesna, A., "The multiresolution analysis of triangle surface meshes with lifting scheme," in [Proceedings of MIRAGE Conference on Computer Vision/Computer Graphics Collaboration Techniques], (2007).

[41] Szczesna, A., "Designing lifting scheme for second generation wavelet-based multiresolution processing of irregular surface meshes," in [Proceedings of Computer Graphics and Visualization], (2008).

[42] Hubeli, A. and Gross, M., "Multiresolution feature extraction from unstructured meshes," in [Proceedings of IEEE Visualization], 287-294 (2001).

[43] Yu, P., Han, X., Ségonne, F., Pienaar, R., Buckner, R. L., Golland, P., Grant, P. E., and Fischl, B., "Cortical surface shape analysis based on spherical wavelet transformation," in [Proceedings of the Computer Vision and Pattern Recognition Workshop], (2006).

[44] Peng, J., Strela, V., and Zorin, D., "A simple algorithm for surface denoising," in [Proceedings of IEEE Visualization], 107-112 (2001).

[45] Faucheur, X. L., Vidakovic, B., Nain, D., and Tannenbaum, A., "Adaptive bayesian shrinkage model for spherical wavelet based denoising and compression of hippocampus shapes," in [Proceedings of the Workshop on Computational Anatomy and Physiology of the Hippocampus], 87-96 (2008).

[46] Roy, M., Foufou, S., and Truchetet, F., "Denoising 3D models with attributes using soft thresholding," in [Proceedings of SPIE Optics East, Wavelet Applications in Industrial Processing], (2004).

[47] Pajarola, R. and Gobbetti, E., "Survey of semi-regular multiresolution models for interactive terrain rendering," The Visual Computer 23(8) (2007).

[48] A. Lee, W. S. and Schröder, P., "Multiresolution mesh morphing," in [Proceedings of ACM SIGGRAPH], 343-350 (1999).

[49] Biermann, H., Kristjansson, D., and Zorin, D., "Approximate boolean operations on free-form solids," in [Proceedings of ACM SIGGRAPH], (2001).

[50] Biermann, H., Martin, I. M., Zorin, D., and Bernardini, F., "Sharp features on multiresolution subdivision surfaces," in [Proceedings of Pacific Graphics], (2001).

[51] Valette, S. and Prost, R., "Wavelet-based progressive compression scheme for triangle meshes: Wavemesh," IEEE Transactions on Visualization and Computer Graphics 10(2), 123-129 (2004).

[52] Chen, R., Luo, X., Zheng, G., and Ling, R., "Multi-resolution compression of meshes based on reverse interpolatory $\sqrt{3}$-subdivision scheme," International Journal of Computer Science and Network Security 6(5A), 100-108 (2006).

[53] El-Leithy, S. T. and Sheta, W. M., "Wavelet-based geometry coding for three dimensional mesh using space frequency quantization," Journal of Computer Science 5(8), 564-569 (2009).

[54] Kammoun, A., Payan, F., and Antonini, M., "Bit allocation for spatio-temporal wavelet coding of animated semi-regular meshes," in [Proceedings of 15th International MultiMedia Modeling Conference (MMM)], (2009).

[55] Wang, K., Lavoué, G., Denis, F., and Baskurt, A., "A comprehensive survey on three-dimensional mesh watermarking," IEEE Transactions on Multimedia 10(8), 1513-1527 (2008).

[56] Cho, W. H., Lee, M. E., Lim, H., and Park, S. Y., "Bit allocation for spatio-temporal wavelet coding of animated semi-regular meshes," in [Proceedings of the International Workshop on Digital Watermarking], 259-270 (2005).

[57] Wang, K., Lavoué, G., Denis, F., and Baskurt, A., "A fragile watermarking scheme for authentication of semi-regular meshes," in [Proceedings of the Eurographics Short Papers], 259-270 (2008).

[58] Wang, K., Lavoué, G., Denis, F., and Baskurt, A., "Hierarchical watermarking of semiregular meshes based on wavelet transform," IEEE Transactions on Information Forensics and Security 3(4), 3657-3660 (2008). 